(2)

\title{
Individualized immunosuppression in transplant patients: potential role of pharmacogenetics
}

This article was published in the following Dove Press journal:

Pharmacogenomics and Personalized Medicine

16 June 2012

Number of times this article has been viewed

\author{
Hamid Abboudi \\ lain AM MacPhee \\ Division of Clinical Sciences, \\ Renal Medicine, St George's, \\ University of London, London, UK
}

\begin{abstract}
The immunosuppressive drugs used to prevent the rejection of transplanted organs have a narrow therapeutic index. Under treatment results in episodes of rejection leading to either damage or loss of the organ. Over immunosuppression increases the risk of infection and malignancy as well as drug specific complications including diabetes mellitus and nephrotoxicity. There is wide variation in the drug dose required to achieve target blood concentrations and there is often dissociation between pharmacokinetics and pharmacodynamics. Currently, immunosuppressive drug treatment is individualized based on a clinical assessment of the risk of rejection or toxicity. Therapeutic drug monitoring is routinely employed for several immunosuppressive drugs. Pharmacogenetics has the potential to complement therapeutic drug monitoring but clinical benefit has yet to be demonstrated. Novel biomarker-based approaches to risk stratification and pharmacodynamic monitoring are under development and are ready for clinical trials.
\end{abstract}

Keywords: CYP3A5, immunosuppression, pharmacogenetics, transplantation

\section{Introduction}

Immunosuppresion is of fundamental importance to the long-term survival of transplanted organs. Over the last 50 years there have been great advances in our knowledge of the immune system and the potential therapeutic targets for pharmacological intervention leading to improved patient and graft survival. Despite this, the rate of allograft loss per year is around 3\%-5\% with major improvements in short term outcomes failing to translate into the same magnitude of benefit in the long term. ${ }^{1}$ As the number of acute rejection episodes has decreased, the complications associated with long-term immunosuppression have become increasingly evident. This has driven research into reducing the toxic effects of immunosuppression. Perhaps the greatest challenge to the clinician is the ever growing demand to tailor therapy to suit individual patient characteristics, and balance the advantages and disadvantages of these treatments.

\section{Overview of immunosuppressive drug treatment}

The immune system can be targeted at several levels. It is always important to remember that any reduction in immune responsiveness to the allograft is accompanied by reduced immunity to infection and malignant disease, in particular DNA viruses such as cytomegalovirus, Epstein-Barr virus, and BK polyomavirus, which causes nephropathy and renal allograft loss.

Prospective study data have indicated that acute rejection is an important predictor of long-term graft function, therefore many interventions have focused on
Correspondence: lain AM MacPhee Division of Clinical Sciences, Renal Medicine, St George's, University of London, Cranmer Terrace, London SWI7 ORE, UK

Tel +442087255035

Fax +442087255036

Email imacphee@sgul.ac.uk 
minimizing acute rejection episodes. Industry-sponsored studies conducted as part of the licensing process for new drugs have tended to focus on the short term outcomes of early acute rejection rate and 1 year graft survival. As a consequence the evidence base for optimal immunosuppressive treatment in the early period after transplantation is strong with much weaker evidence to indicate optimal long-term therapy.

\section{Induction immunosuppressive therapy}

Episodes of acute rejection occur most common in the early period after transplantation and are rare beyond 3 months after transplantation with most immunosuppressive regimens. This has been achieved largely through the use of induction therapy in the peritransplantation period (Table 1). The term "induction therapy" refers to potent agents given during the early period after transplantation that are not continued long term. In the United States between 1998 and 2007, a total of $78 \%$ of renal transplant recipients received antibody induction therapy. ${ }^{2}$ Induction agents can be grouped into drugs causing cell depletion (lytic induction) and drugs blocking cell surface receptors without depletion (nonlytic induction). In general, lytic induction agents are more potent with lower rates of acute rejection but higher incidence of infectious and malignant complications. The only nonlytic induction agent in widespread use is basiliximab, a monoclonal antibody specific for the alpha chain of the interleukin (IL)-2 receptor expressed on activated T-lymphocytes. Frequently used lytic induction agents include rabbit antithymocyte globulin (ATG; Thymoglobulin ${ }^{\circledR}$; SangStat Medical Corporation, Fremont, CA), a lymphocyte depleting polyclonal antibody. The recent introduction of alemtuzumab, a humanized

Table I Induction immunosuppression drugs

\begin{tabular}{lll}
\hline Drug & Mechanism of action & Side effects \\
\hline ATG/ALG & $\begin{array}{l}\text { Complement mediated lysis } \\
\text { Opsonization and clearance } \\
\text { blockade of cell surface } \\
\text { receptors }\end{array}$ & $\begin{array}{l}\text { Agranulocytosis } \\
\text { Thrombocytopenia } \\
\text { Cytokine release } \\
\text { syndrome }\end{array}$ \\
$\begin{array}{l}\text { Basiliximab } \\
\text { (anti-CD25 }\end{array}$ & Blockade of IL-2 receptor & \\
Alemtibody) & & \\
OKT3 & T and B lymphocyte depletion & Cytokine release \\
& Complement-mediated lysis & Cytokine release \\
& $\begin{array}{l}\text { opsonization and clearance } \\
\text { blockade of CD3 receptor }\end{array}$ & syndrome \\
\hline
\end{tabular}

Abbreviations: ATG, antithymocyte globulin; ALG, antilymphocyte globulin; IL-2, interleukin-2; OKT3, monoclonal anti-CD3 antibody. monoclonal antibody that is specific for CD52 and depletes T- and B-lymphocytes, natural killer cells, and monocytes has seen increasing use. One large, prospective, randomized controlled trial showed that despite superior prevention of biopsy confirmed acute rejection at 6, 12, and 36 months compared to basiliximab, alemtuzumab was associated with

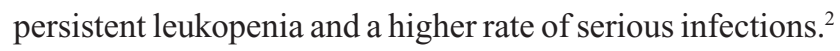
Late episodes of acute rejection in the period 3-12 months after transplantation are more common in alemtuzumabtreated patients than with other regimens. ${ }^{2}$ Normally one of these induction agents is also combined with either a short course of corticosteroid therapy or longer term corticosteroid use as well as introduction of the maintenance regimen described below. Given the advantages and disadvantages of all the agents available and the multiple different regimens, it is still not clear which is the best formula. ATG is the most commonly used agent and is very potent, whereas basiliximab is less potent but offers fewer complications. The choice of regimen depends on an assessment of the risks of rejection and complications of over immunosuppression.

\section{Maintenance immunosuppressive therapy}

Maintenance immunosuppression may be best achieved with a combination approach although some clinicians prefer calcineurin inhibitor (CNI) monotherapy. Combination therapy aims to minimize the side effects of any single drug whilst maintaining adequate overall immunosuppression. The principle is to target multiple levels of lymphocyte activation and inflammation. This is achieved by combining corticosteroids with a CNI (cyclosporin or tacrolimus) and an antiproliferative agent such as azathioprine or mycophenolate (Table 2). Several factors such as degree of human leukocyte antigen incompatibility, presence of human leukocyte antigen antibody, recipient ethnic group, previous transplants, and history of previous acute rejection influence the choice and dosage of specific maintenance agents, based on risk of acute rejection or toxicity. The potential for use of more sophisticated markers for risk stratification is discussed below.

\section{Maintenance therapies widely used in solid organ transplant recipients Azathioprine}

Azathioprine was developed in the early 1960s and functions as an antimetabolite to decrease lymphocyte DNA and RNA synthesis. A more recently identified mechanism of action is the inhibition of intracellular signaling following activation 
Table 2 Maintenance immunosuppression drugs

\begin{tabular}{|c|c|c|}
\hline Drug & Mechanism of action & Side effects \\
\hline \multirow[t]{4}{*}{ Corticosteroids } & Blocks transcription of cytokine genes, IL-I, IL-2, IL-3, IL-5, IL-6, tumor & Hypertension \\
\hline & necrosis factor- $\alpha$, and interferons & Glucose intolerance \\
\hline & & Dyslipidemia \\
\hline & & Osteoporosis \\
\hline \multirow[t]{4}{*}{ Cyclosporin } & Inhibits IL-2 production, stimulates TGF- $\beta$ production & Nephrotoxicity \\
\hline & & Hypertension \\
\hline & & Dyslipidemia \\
\hline & & Glucose intolerance \\
\hline \multirow[t]{4}{*}{ Tacrolimus } & Inhibits IL-2 production & Nephrotoxicity \\
\hline & & Hypertension \\
\hline & & Dyslipidemia \\
\hline & & Glucose intolerance \\
\hline \multirow[t]{2}{*}{ Azathioprine } & Inhibits purine synthesis and blocks DNA and RNA synthesis & Myelosuppression \\
\hline & Inhibits CD28 signaling & \\
\hline \multirow[t]{2}{*}{ Mycohphenolate mofetil } & Blocks de novo pathway of purine synthesis (selective for lymphocytes) & Diarrhea and gastrointestinal upset \\
\hline & and blocks glycosylation & Myelosuppression \\
\hline \multirow[t]{4}{*}{ Sirolimus/everolimus } & Blocks IL-2-induced cell cycle progression & Hyperlipidemia \\
\hline & & Thrombocytopenia \\
\hline & & Impaired wound healing \\
\hline & & Oral ulcers \\
\hline Belatacept & Blocks costimulation by $C D 28$ & PTLD in EBV naïve patients \\
\hline
\end{tabular}

Abbreviations: EBV, Epstein-Barr virus; IL, interleukin; PTLD, posttransplant lymphoproliferative disorder; TGF, transforming growth factor.

of the CD28 pathway. ${ }^{3}$ Side effects of azathioprine include leukopenia, thrombocytopenia, gastrointestinal disturbance, hepatitis, cholestasis, and alopecia. Consequently, blood counts need to be monitored regularly as myelosuppression can be potentially fatal. Azathioprine is free from any important metabolic or cardiovascular toxicities.

\section{Corticosteroids}

Corticosteroids have long been the main therapeutic agent during induction and maintenance of immunosuppression. Their mechanism of action involves inhibiting a broad range of cytokines, including IL-1 and IL-6 production by macrophages as well as all stages of T-lymphocyte activation. Corticosteroids are also very effective when used at high doses to treat episodes of acute rejection. The long-term complications associated with corticosteroid use are well documented and include hypertension, diabetes mellitus, cataracts, osteoporosis, hyperlipidemia, and infections. ${ }^{4}$ Corticosteroid-free immunosuppression is gaining popularity given that a large proportion of transplant loss is related to cardiovascular mortality and/or fatal infectious complications and that the long-term use of corticosteroids contributes to an adverse cardiovascular risk factor profile. With progressive improvement in the potency of the other immunosuppressive drugs, corticosteroid-avoidance or minimization can often be achieved. A large reduction in the number of patients being discharged on corticosteroids has reflected the trend of corticosteroid-free treatment or at least minimal corticosteroid use. ${ }^{5}$ Benefits obtained include decreased blood cholesterol and triglyceride concentrations, decreased blood pressure medication requirement, ${ }^{6-8}$ and lower rates of new onset diabetes after transplantation (NODAT), ${ }^{9,10}$ cardiovascular events, and metabolic syndrome. ${ }^{11,12}$ Despite the advantages of corticosteroid-free immunosuppression, acute rejection remains lower in patients receiving corticosteroid-containing regimens. ${ }^{5}$

\section{Calcineurin inhibitors (CNI) Cyclosporin}

Introduced in the 1980s, cyclosporin binds to the intracellular molecule cyclophilin and the complex inhibits the phosphatase calcineurin. This in turn prevents the dephosphorylation of nuclear factor of activated T-lymphocytes with consequent inhibition of IL-2 synthesis. IL-2 is a key cytokine in driving the proliferation of T-lymphocytes. On introduction of cyclosporin, 1 year survival rates for renal allografts improved from approximately $60 \%$ to between $80 \%$ and $90 \%{ }^{13}$ Cyclosporin is associated with nephrotoxicity due to vasoconstriction causing ischemia and direct toxic effects on renal tubular epithelial cells, which has driven attempts to find alternative drugs. ${ }^{14}$ While it was hoped that a more recently introduced CNI, tacrolimus, would be less nephrotoxic, this is not clear from the available published data. ${ }^{15}$ In one report, nearly all 99 recipients of kidneypancreas transplants who received cyclosporin-based immunosuppression had nephrotoxicity 10 years after transplantation, 
with the median onset of the first lesion at 6 months. ${ }^{16}$ However, histological changes ascribed to CNI toxicity have been questioned recently with similar changes found in patients not treated with CNIs. ${ }^{17}$ Its side effect profile has led to several studies aiming to minimize CNI use. One such study was the CAESAR trial, ${ }^{18}$ which compared graft function 12 months postoperatively among patients randomly assigned to one of three groups; daclizumab (a CD25 antibody that has now been withdrawn from the market for commercial reasons), mycophenolate mofetil (MMF), corticosteroid, and low-dose cyclosporin (target trough concentrations of 50-100 ng/mL), weaned from month 4 and withdrawn by month 6 ; daclizumab, MMF, corticosteroid, and low-dose cyclosporin; or MMF, corticosteroid, and standard-dose cyclosporin. The main aim was to determine if withdrawing cyclosporin would provide appropriate immunosuppression but this was not the case, with higher rates of acute rejection episodes in the cyclosporin withdrawal group. However, the low-dose group provided similar if not better results at 12 months compared to the standard group, which heralded the potential for CNIminimizing therapy.

\section{Tacrolimus}

The other currently licensed CNI is tacrolimus, a macrolide structurally related to the antibiotic erythromycin. It is more potent than cyclosporin, with some data ${ }^{19}$ suggesting improved graft survival; but this comes at the expense of higher rates of NODAT and neurological symptoms including tremor and paresthesia. As mentioned above, tacrolimus is nephrotoxic, ${ }^{19}$ but it is associated with a lower incidence of hyperlipidemia and hypertension than cyclosporin. ${ }^{20}$ This is important because cardiovascular disease is the most common cause of premature death in transplant recipients. ${ }^{21}$

The most widely used immunosuppressive regimen for renal transplantation is based on the results of the ELITE-Symphony trial, ${ }^{22}$ in which 1645 renal transplant recipients were randomized to receive either standard-dose cyclosporin, MMF, and corticosteroids, or daclizumab induction, $\mathrm{MMF}$, and corticosteroids, in combination with low-dose cyclosporin, low-dose tacrolimus, or low-dose sirolimus. Treatment for 12 months with a regimen containing daclizumab, MMF, corticosteroids, and low-dose tacrolimus provided adequate immunosuppression compared to the other three treatment groups as evidenced by better renal function and renal allograft survival, and lower rates of acute rejection. In addition, the low-dose tacrolimus regimen provided better allograft survival than regimens with standard-dose cyclosporin or low-dose sirolimus.
This did come at the expense of a higher incidence of NODAT than with the other regimens and a higher incidence of diarrhea, which may reflect excessive MMF dosing. This study has paved the way for lower doses of CNIs while still providing effective immunosuppression with a concomitant reduction in the risks associated with these medications. ${ }^{22}$

Other authors have looked at eliminating the adverse effects of CNIs by discontinuing their use in immunologically low-risk patients, which has been associated with favorable long-term results. ${ }^{23,24}$ Bakker et al showed that conversion to a CNI-free immunosuppressive regimen 3 months after renal transplantation improved allograft function, reduced the need for cardiovascular risk factor controlling medication, and reduced the incidence of chronic allograft nephropathy (now referred to as interstitial fibrosis with tubular atrophy). ${ }^{23}$

\section{Mammalian target of rapamycin (mTOR) inhibitors}

The mammalian target of rapamycin (mTOR) inhibitors block the response of T-lymphocytes to IL-2 and have additional antiproliferative effects. There are two drugs currently licensed in this class; sirolimus, a macrolide, and everolimus, which was derived by adding a hydroxyethyl group to sirolimus. Sirolimus has a long-elimination half-life of 60 hours allowing once-daily dosing, ${ }^{25}$ in comparison to the shorter half-life of everolimus of 18-35 hours requiring twice-daily dosing. ${ }^{26}$ When initially introduced, it was hoped that the mTOR inhibitors would provide an alternative to the CNIs for use in de novo transplants that was not nephrotoxic and did not cause hypertension. However, they are not quite as potent as the CNIs resulting in higher rates of acute rejection, while the antiproliferative effects include impaired would healing, increased incidence of lymphoceles, ${ }^{27,28}$ and delayed recovery from acute tubular necrosis. The inhibition of the scarring process may be of benefit in reducing interstitial fibrosis and tubular atrophy after the early transplant phase and a number of regimens now involve initial use of a CNI with switch to an mTOR inhibitor after the initial 3-month period. The mTOR inhibitors can be used along with CNIs and this is the basis for the license for everolimus. A potential problem with this approach is the potentiation of CNI nephrotoxicity through inhibition of the drug transporter P-glycoprotein (P-gp) by sirolimus leading to increased intracellular drug concentration. ${ }^{29,30}$ Hypertriglyceridemia, hypercholesterolemia, microcytic anemia, and thrombocytopenia are also problems.

The mTOR inhibitors have anticancer properties. ${ }^{31}$ Interestingly everolimus has been extensively studied in 
the field of oncology as a potential treatment for renal cell carcinomas, neuroendocrine tumors of the intestine, mantle cell lymphoma, and sarcomas.

Barrier to absorption of the CNIs and mTOR inhibitors The oral bioavailability of the CNIs and mTOR inhibitors is only in the order of $20 \%-30 \%$. This is in part due to the active barrier to absorption posed through first pass metabolism in the intestine and liver by the enzymes cytochrome P450 3A4 and 3A5 (CYP3A4, CYP3A5) and by the drug transporter P-gp (the product of the $A B C B 1$ gene). A significant proportion of the variation in oral bioavailability between individuals and drug interactions are based on the effectiveness of this barrier. ${ }^{32}$

\section{Mycophenolate}

The active drug mycophenolic acid is available as a morpholinoethyl ester, MMF or as enteric-coated mycophenolate sodium. Mycophenolic acid inhibits the enzyme inosine monophosphate dehydrogenase (IMPDH) and impairs B- and T-lymphocyte proliferation, sparing other rapidly dividing cells. MMF is a more potent adjunct therapy used along with CNI than azathioprine. ${ }^{33}$ While acute rejection in the early period after transplantation is reduced, impact on longer term outcomes is less certain although there are some positive data. ${ }^{34}$

\section{Belatacept}

Belatacept is the most recently introduced immunosuppressive drug. It is a fusion protein between a genetically modified CD152 (CTLA4) domain and a human immunoglobulin $\mathrm{G}$ domain. It blocks costimulation of T-lymphocytes via the CD28 pathway and requires intravenous administration, more frequently during the early period after transplantation and then monthly. When compared to cyclosporin, acute rejection rates were higher but there was less chronic damage on protocol biopsies. An increased incidence of posttransplant lymphoproliferative disorder in Epstein-Barr virus naïve patients is an important concern. The pharmacokinetics of belatacept are predictable with limited variation between individuals, unlike the small molecule immunosuppressive drugs. ${ }^{35}$

\section{Specific inhibition of antibody production}

Acute and chronic antibody-mediated rejection are amongst the most difficult complications of transplantation to treat. Removal of donor-specific antibodies to allow transplantation in immunologically sensitized patients is another challenging area that is a focus of current research. The most widely used anti-B-lymphocyte agent is rituximab, a chimeric antibody to the CD20 molecule that is expressed on B-lymphocytes but not mature plasma cells or pro-B-lymphocytes. It has been used as an induction agent, along with intravenous immunoglobulin and antibody removal by immunoabsorption or plasma exchange, to reduce the rate of antibody resynthesis in antibody incompatible transplantation. There are some data to suggest efficacy in acute antibody mediated rejection and trials of efficacy in chronic antibody mediated rejection are underway. ${ }^{36}$ The proteasome inhibitor bortezomib depletes mature plasma cells offering direct targeting of the source of antibody production. There is evidence of bortezomib efficacy in acute antibody mediated rejection. ${ }^{37}$ One of the primary mechanisms of antibody mediated damage of vascular endothelium is through the activation of complement. Eculizumab is an antibody specific for the C5 component of complement and inhibits the formation of the membrane attack complex preventing complement-mediated damage to cells. It has been used for both the prevention and treatment of acute antibody mediated rejection. ${ }^{38}$

\section{Interindividual variability of transplant rejection and potential for individualized immunosuppression Identification of patients at high risk of rejection or toxicity}

In recent years the number of acute rejection episodes has decreased, therefore the main challenges are to reduce long-term toxicity and transplant damage due to chronic rejection, with an increasingly recognized role for chronic antibody mediated rejection. Multiple factors predispose chronic allograft damage, including the condition of the donor organ, ischemia reperfusion injury, acute or chronic immunological rejection, drug toxicity, hypertension, and infection. ${ }^{39}$ Identification of risk for acute rejection in both the short and long term would allow individualization of immunosuppressive treatment. An area of substantial recent activity has been the identification of patients with complete or partial immunological tolerance to transplantation to allow safe minimization of immunosuppression. The initial phase of this research was based on studies of noncompliant patients who continued to have well-functioning grafts after complete immunosuppression withdrawal (operational tolerance). ${ }^{40,41}$ International consortia have identified a cross platform biomarker signature for operational tolerance 
including expansion of peripheral blood B-lymphocytes, absence of donor specific antibodies, donor specific hyporesponsiveness of $\mathrm{CD}^{+}{ }^{+} \mathrm{T}$-lymphocytes, and a high ratio of FoxP3 to $\alpha-1,2-$ mannosidase gene expression in peripheral blood. ${ }^{40,41}$ Attempts are now underway to qualify clinically useful biomarkers based on these data to allow targeted immunosuppression minimization or withdrawal.

\section{Monitoring of response to immunosuppressive therapy}

Assays of response to immunosuppression are close to clinical application, or so called pharmacodynamic monitoring. These assays are based on measurement of the degree of inhibition of target enzymes or signaling pathways. Patients with significant residual expression of nuclear factor of activated T-lymphocyte regulated genes on treatment with $\mathrm{CNI}$ have a higher incidence of acute rejection than those with potent suppression, but are less susceptible to infection. This information could allow either use of an alternative agent or higher CNI blood concentrations. ${ }^{42}$ Similarly, assays of IMPDH activity have been proposed as a strategy for identification of response to MMF but there is wide variation in response between individuals, which will make clinical application challenging. ${ }^{43}$ Measurement of IMPDH activity in CD4+ ${ }^{+}$-lymphocytes represents a potential refinement of this approach. ${ }^{44} \mathrm{~A}$ variety of markers of inhibition of lymphocyte activation have been validated for testing in clinical trials. ${ }^{45}$ There is a commercially available assay (Immuknow ${ }^{\text {TM}}$; Cylex, Columbia, MD) that measures inhibition of the proliferation of $\mathrm{CD}^{+}{ }^{+} \mathrm{T}-$ lymphocytes in response to a mitogen. While rejection rates were higher and infection rates were lower, the wide variation within the groups again limits the clinical utility of this assay, ${ }^{46}$ a common flaw for all of the pharmacodynamic assays. Prospective data on the impact of pharmacodynamic monitoring on outcomes are awaited.

\section{Potential for pharmacogenetics in clinical decision making}

Therapeutic drug monitoring (TDM) is routinely used to achieve therapeutic blood concentrations of cyclosporin, tacrolimus, sirolimus, and everolimus. TDM has been used for MMF by some centers, although this is controversial. ${ }^{47}$ Genetic factors influence pharmacokinetics and pharmacogenetic testing has potential as an adjunct to TDM. Genetic polymorphisms in drug targets, drug metabolizing enzymes, and drug transporters have been identified as potential targets for developing a pharmacogenetic strategy to individualize initial drug choice and dose. ${ }^{32}$ This is most likely to be useful where early achievement of target blood concentrations is important, for prevention of acute rejection, avoidance of significant toxicity, or when there may be some delay using the reactive strategy of TDM. This is the case for cyclosporin, ${ }^{48}$ tacrolimus, ${ }^{49}$ and MMF. ${ }^{50}$ Another area of potential utility is for drugs with a long-elimination half-life such as sirolimus, where the prolonged time to reach steady state after dose adjustments, in response to blood concentrations outside the target range, may result in significant delay in achieving target blood concentrations. Genotyping for mutations in the thiopurine-S-methyltransferase gene that metabolizes azathioprine has been widely adopted in some therapeutic areas but not transplantation. ${ }^{51}$ This may be due to frequent blood count monitoring in transplant recipients following the introduction of azathioprine allowing the avoidance of severe myelotoxicity. Potentially useful pharmacogenetic strategies to guide the prescription of immunosuppressive drugs are summarized in Table 3.

Table 3 Summary of potential pharmacogenetic strategies to guide immunosuppressive drug prescribing

\begin{tabular}{|c|c|c|c|}
\hline Drug & Genotypes & Evidence & Ref \\
\hline \multirow[t]{4}{*}{ Tacrolimus } & CYP $3 A 5 * 1 / * 3$ & Tested in a randomized controlled trial with earlier achievement of target blood & 58 \\
\hline & & concentrations but no influence on clinical outcome. & 59 \\
\hline & CYP $3 A 4 * 22$ & May provide additional predictive value to algorithms in combination with CYP3A5*I/*3. & 60 \\
\hline & $P O R^{*} 28$ & & 61 \\
\hline Sirolimus & СYРЗА5*I/*3 & Impact on dose-normalized blood concentrations but not yet tested in a clinical trial. & $67-69$ \\
\hline \multirow[t]{2}{*}{ Cyclosporin } & CYP $3 A 5 * 1 / * 3$ & Combination of genotypes may predict drug exposure but not yet replicated. & 66 \\
\hline & CYP $3 A 4 * 22$ & & \\
\hline \multirow[t]{2}{*}{ Azathioprine } & TPMT & Predicts individuals likely to develop severe myelotoxicity. Limited adoption & 51 \\
\hline & & in transplantation with no intervention study data. & \\
\hline \multirow[t]{4}{*}{ Mycophenolate } & UGTIA9 & Predicts individuals likely to be under-exposed to MPA with increased risk of rejection. & 71 \\
\hline & IMPDHI & Predicts individuals at increased risk of rejection. & 72 \\
\hline & & Genetic component to variability in MPA exposure is much smaller than other factors, & \\
\hline & & limiting the potential of pharmacogenetics. & \\
\hline
\end{tabular}

Abbreviations: IMPDH, inosine monophosphate dehydrogenase; MPA, mycophenolic acid. 


\section{Tacrolimus}

The drug for which the potential application of pharmacogenetics has been most extensively tested is tacrolimus. Tacrolimus pharmacokinetics are influenced by a variety of factors including hepatic dysfunction, previous hepatitis $\mathrm{C}$ virus infection, time after transplantation, patient age, donor liver characteristics, recipient ethnic group, hematocrit and serum albumin concentrations, diurnal rhythm, food administration, corticosteroid dosage, diarrhea, and CYP isoenzyme and P-gp expression. ${ }^{52,53} \mathrm{~A}$ very crude form of pharmacogenetics that is applied in some centers is the administration of increased doses of tacrolimus to individuals who are genetically sub-Saharan African (Black). ${ }^{54}$ Investigation of possible genetic factors underlying the high tacrolimus dose requirement in Black patients led to the identification of CYP3A5 expresser status as a key determinant of the oral bioavailability of tacrolimus. ${ }^{55,56}$ People are either functional expressers or nonexpressers of the metabolic enzyme CYP3A5, a key component of the first pass barrier to drug absorption. Expression is determined by single nucleotide polymorphisms (SNPs) in the CYP $3 A 5$ gene. Individuals with at least one wild-type $C Y P 3 A 5^{*} 1$ allele are functional expressers and homozygotes for the mutant $C Y P 3 A 5^{*} 3$ allele are functional nonexpressers. CYP3A5 expressers take longer to achieve target blood tacrolimus concentrations after transplantation, require higher doses of tacrolimus, and experience episodes of acute rejection earlier than nonexpressers. ${ }^{57}$ An important caveat is that in immunosuppressive regimens based on potent induction therapy and other adjunctive agents, early attainment of target blood concentrations of tacrolimus may not be critical, resulting in absence of observed influence on the incidence of acute rejection in some studies. ${ }^{58}$ A multicenter study in France randomized renal transplant recipients at relatively low risk of rejection to receive either a standard initial tacrolimus dose of $0.2 \mathrm{mg} / \mathrm{kg}$ or to a dose based on genotype: $0.3 \mathrm{mg} / \mathrm{kg}$ for CYP3A5 expressers or $0.15 \mathrm{mg} / \mathrm{kg}$ for nonexpressers. The majority of patients were given high doses of ATG as induction therapy with high doses of MMF. Significantly more patients in the genetically predicted group achieved the target blood concentration within 3 days of starting treatment with tacrolimus than in the standard dose group $(43.2 \%$ versus $29.1 \%, P=0.03) .{ }^{59}$ While this study achieved a modest improvement in the number of patients with blood concentrations within the target range, over half of the patients were still outside the range and there was no difference between the groups in incidence of acute rejection or toxicity. A key factor in the absence of positive pharmacodynamic endpoints was the delay in introduction of tacrolimus until 7 days after transplantation.

Addition of other genotypes into a more complex polygenic algorithm may allow refinement of the genetic prediction. Other candidate genotypes that have been shown to have a significant influence on tacrolimus pharmacokinetics are $A B C B 1$ (encoding P-gp), ${ }_{55} C Y P 3 A 4 * 22^{60}$ and $P 450$ oxidoreductase $* 28 .{ }^{61}$ It may be useful to combine genetic and demographic factors ${ }^{62}$ but this algorithm remains to be tested on another cohort of patients. There has been recent interest in the pregnane $\mathrm{X}$ receptor that regulates the expression of cytochrome P450 and P-gp and its genetic variants as potential predictors of interindividual differences in drug concentrations. ${ }^{63}$ The extent of pregnane $\mathrm{X}$ receptor effects on immunosuppression in transplant patients has not been investigated.

\section{Cyclosporin}

Cyclosporin pharmacokinetics are, among other factors, influenced by body weight and concomitant prednisolone exposure, and genetic markers are yet to be identified that explain the interindividual variability. ${ }^{64}$ While $C Y P 3 A 5$ genotype alone is not predictive of cyclosporin dose requirements, ${ }^{65}$ addition of the $C Y P 3 A 4 * 22$ genotype to the algorithm provides a better prediction but with a large degree of overlap between the genotype groups. ${ }^{66}$

\section{Sirolimus and everolimus}

The influence of CYP3A5 expression on the pharmacokinetics of sirolimus is less well studied. However, when prescribed without a CNI, the oral bioavailability of sirolimus is lower in CYP3A5 expressers ${ }^{67}$ and consequently takes longer to achieve target blood concentrations than nonexpressers. ${ }^{68}$ Furthermore, expressers require significantly more sirolimus to achieve adequate blood trough concentrations. ${ }^{69}$ There was no significant influence of CYP $3 A 5$ genotype on the pharmacokinetics of everolimus. ${ }^{70}$

\section{MMF}

MMF dose-prediction may be influenced by the expression of SNPs, which affect the metabolism, transport, and target enzyme for mycophenolic acid. The UGT1A9 and IMPDHI genes contain SNPs which are predictive of acute rejection. ${ }^{71,72}$ However, there are no data on the use of these genotypes in guiding MMF dosing. 


\section{Drug transporters}

Drug transporters may be useful in developing a pharmacogenetic strategy for immunosuppression. The transporters influence toxicity, metabolism, and elimination of drugs within cells and are subject to genetic variation. One such transporter is $\mathrm{P}$-gp, which is encoded by the $A B C B 1$ gene. In theory, higher concentrations of P-gp on T-lymphocytes will lead to reduced intracellular concentrations of immunosuppressant drugs. It has been demonstrated that $A B C B 1$ polymorphisms influence cyclosporin intracellular concentration; individuals homozygous for wild-type alleles presented a 1.8-fold decreased cyclosporin intracellular concentration $(P=0.04)$, compared to those with the mutant genotype. ${ }^{73}$ Similar observations have been published for tacrolimus. ${ }^{74}$

\section{Conclusion}

Heterogeneity in the pharmacokinetics and pharmacodynamics of immunosuppressive agents combined with their narrow therapeutic index renders them a difficult class of drug to prescribe. Selection of appropriate immunosuppressive regimens based on predicted risk of rejection and toxicity is only possible on a relatively crude basis at present but there is potential for refinement based on immunological and pharmacodynamic assays. We await the qualification of these potential biomarkers in clinical studies. In the meantime we are left with optimizing immunosuppressive drug prescribing based on pharmacokinetic measurements. Pharmacogenetic testing has potential as an adjunct to TDM in optimizing drug exposure. The only candidate pharmacogenetic strategy that has been tested in a clinical trial is CYP $3 A 5$ and tacrolimus but this strategy has not yet demonstrated definite clinical benefit. Refinement of dosing algorithms for tacrolimus based on all of the genes known to be involved in pharmacokinetics and demographic factors, followed by testing in a clinical trial with sufficient statistical power to demonstrate reduction in the incidence of rejection or toxicity, would be a logical next step. Further testing of pharmacogenetic strategies based on the CYP3A5 genotype is warranted for sirolimus. Clinical trials of other pharmacogenetic candidates would be premature at present. Genome wide association studies currently underway may identify further candidate genes.

\section{Disclosure}

The authors report no conflicts of interest in this work.

\section{References}

1. Lodhi SA, Lamb KE, Meier-Kriesche HU. Solid organ allograft survival improvement in the United States: the long-term does not mirror the dramatic short-term success. Am J Transplant. 2011;11:1226-1235.
2. Hanaway MJ, Woodle ES, Mulgaonkar S, et al. Alemtuzumab induction in renal transplantation. $N$ Engl J Med. 2011;364:1909-1919.

3. Tiede I, Fritz G, Strand S, et al. CD28-dependent Rac1 activation is the molecular target of azathioprine in primary human CD4+ T lymphocytes. J Clin Invest. 2003;111:1133-1145.

4. Schacke H, Docke WD, Asadullah K. Mechanisms involved in the side effects of glucocorticoids. Pharmacol Ther. 2002;96:23-43.

5. Luan FL, Steffick DE, Gadegbeku C, Norman SP, Wolfe R, Ojo AO. Graft and patient survival in kidney transplant recipients selected for de novo steroid-free maintenance immunosuppression. Am JTransplant. 2009;9:160-168.

6. Vincenti F, Schena FP, Paraskevas S, Hauser IA, Walker RG, Grinyo J. A randomized, multicenter study of steroid avoidance, early steroid withdrawal or standard steroid therapy in kidney transplant recipients. Am J Transplant. 2008;8:307-316.

7. Smak Gregoor PJ, de Sevaux RG, Ligtenberg G, et al. Withdrawal of cyclosporine or prednisone six months after kidney transplantation in patients on triple drug therapy: a randomized, prospective, multicenter study. J Am Soc Nephrol. 2002;13:1365-1373.

8. Rostaing L, Cantarovich D, Mourad G, et al. Corticosteroid-free immunosuppression with tacrolimus, mycophenolate mofetil, and daclizumab induction in renal transplantation. Transplantation. 2005;79:807-814.

9. Woodle ES, First MR, Pirsch J, Shihab F, Gaber AO, Van VP. A prospective, randomized, double-blind, placebo-controlled multicenter trial comparing early ( 7 day) corticosteroid cessation versus longterm, low-dose corticosteroid therapy. Ann Surg. 2008;248:564-577.

10. Kumar MS, Heifets M, Moritz MJ, et al. Safety and efficacy of steroid withdrawal two days after kidney transplantation: analysis of results at three years. Transplantation. 2006;81:832-839.

11. Arnol M, de Mattos AM, Chung JS, Prather JC, Mittalhenkle A, Norman DJ. Late steroid withdrawal and cardiovascular events in kidney transplant recipients. Transplantation. 2008;86:1844-1848.

12. Rike AH, Mogilishetty G, Alloway RR, et al. Cardiovascular risk, cardiovascular events, and metabolic syndrome in renal transplantation: comparison of early steroid withdrawal and chronic steroids. Clin Transplant. 2008;22:229-235.

13. European Multicentre Trial Group. Cyclosporin in cadaveric renal transplantation: one-year follow-up of a multicentre trial. Lancet. 1983;2:986-989.

14. Naesens M, Kuypers DR, Sarwal M. Calcineurin inhibitor nephrotoxicity. Clin J Am Soc Nephrol. 2009;4:481-508.

15. Rowshani AT, Scholten EM, Bemelman F, et al. No difference in degree of interstitial Sirius red-stained area in serial biopsies from area under concentration-over-time curves-guided cyclosporine versus tacrolimus-treated renal transplant recipients at one year. J Am Soc Nephrol. 2006;17(1):305-312.

16. Nankivell BJ, Borrows RJ, Fung CL, O’Connell PJ, Chapman JR, Allen RD. Calcineurin inhibitor nephrotoxicity: longitudinal assessment by protocol histology. Transplantation. 2004;78:557-565.

17. Snanoudj R, Royal V, Elie C, et al. Specificity of histological markers of long-term CNI nephrotoxicity in kidney-transplant recipients under lowdose cyclosporine therapy. Am J Transplant. 2011;11:2635-2646.

18. Ekberg H, Grinyo J, Nashan B, et al. Cyclosporine sparing with mycophenolate mofetil, daclizumab and corticosteroids in renal allograft recipients: the CAESAR Study. Am J Transplant. 2007;7:560-570.

19. Webster AC, Woodroffe RC, Taylor RS, Chapman JR, Craig JC. Tacrolimus versus ciclosporin as primary immunosuppression for kidney transplant recipients: meta-analysis and meta-regression of randomised trial data. BMJ. 2005;331:810.

20. Artz MA, Boots JM, Ligtenberg G, et al. Improved cardiovascular risk profile and renal function in renal transplant patients after randomized conversion from cyclosporine to tacrolimus. J Am Soc Nephrol. 2003;14:1880-1888.

21. Sarnak MJ, Levey AS, Schoolwerth AC, et al. Kidney disease as a risk factor for development of cardiovascular disease: a statement from the American Heart Association Councils on Kidney in Cardiovascular Disease, High Blood Pressure Research, Clinical Cardiology, and Epidemiology and Prevention. Circulation. 2003;108:2154-2169. 
22. Ekberg H, Tedesco-Silva H, Demirbas A, et al. Reduced exposure to calcineurin inhibitors in renal transplantation. $N$ Engl J Med. 2007;357:2562-2575.

23. Bakker RC, Hollander AA, Mallat MJ, Bruijn JA, Paul LC, de Fijter JW. Conversion from cyclosporine to azathioprine at three months reduces the incidence of chronic allograft nephropathy. Kidney Int. 2003;64: 1027-1034.

24. Joss N, Rodger RS, McMillan MA, Junor BJ. Randomized study comparing cyclosporine with azathioprine one year after renal transplantation-15-year outcome data. Transplantation. 2007;83: 582-587.

25. MacDonald A, Scarola J, Burke JT, Zimmerman JJ. Clinical pharmacokinetics and therapeutic drug monitoring of sirolimus. Clin Ther. 2000;22(Suppl B):B101-B121.

26. Kahan BD, Wong RL, Carter C, et al. A phase I study of a 4-week course of SDZ-RAD (RAD) quiescent cyclosporine-prednisone-treated renal transplant recipients. Transplantation. 1999;68:1100-1106.

27. Dean PG, Lund WJ, Larson TS, et al. Wound-healing complications after kidney transplantation: a prospective, randomized comparison of sirolimus and tacrolimus. Transplantation. 2004;77:1555-1561.

28. Langer RM, Kahan BD. Incidence, therapy, and consequences of lymphocele after sirolimus-cyclosporine-prednisone immunosuppression in renal transplant recipients. Transplantation. 2002;74:804-808.

29. Pascual M, Theruvath T, Kawai T, Tolkoff-Rubin N, Cosimi AB Strategies to improve long-term outcomes after renal transplantation. N Engl J Med. 2002;346:580-590.

30. Anglicheau D, Pallet N, Rabant M, et al. Role of P-glycoprotein in cyclosporine cytotoxicity in the cyclosporine-sirolimus interaction. Kidney Int. 2006;70:1019-1025.

31. Campistol JM, Eris J, Oberbauer R, et al. Sirolimus therapy after early cyclosporine withdrawal reduces the risk for cancer in adult renal transplantation. J Am Soc Nephrol. 2006;17:581-589.

32. Ekbal NJ, Holt DW, Macphee IA. Pharmacogenetics of immunosuppressive drugs: prospect of individual therapy for transplant patients Pharmacogenomics. 2008;9:585-596.

33. Halloran P, Mathew T, Tomlanovich S, Groth C, Hooftman L, Barker C. Mycophenolate mofetil in renal allograft recipients: a pooled efficacy analysis of three randomized, double-blind, clinical studies in prevention of rejection. The International Mycophenolate Mofetil Renal Transplant Study Groups. Transplantation. 1997;63:39-47.

34. Ojo AO, Meier-Kriesche HU, Hanson JA, et al. Mycophenolate mofetil reduces late renal allograft loss independent of acute rejection. Transplantation. 2000;69:2405-2409.

35. Vincenti F, Charpentier B, Vanrenterghem Y, et al. A phase III study of belatacept-based immunosuppression regimens versus cyclosporine in renal transplant recipients (BENEFIT study). Am JTransplant. 2010;10: 535-546.

36. Clatworthy MR. Targeting B cells and antibody in transplantation. Am J Transplant. 2011;11:1359-1367.

37. Walsh RC, Brailey P, Girnita A, et al. Early and late acute antibodymediated rejection differ immunologically and in response to proteasome inhibition. Transplantation. 2011;91:1218-1226.

38. Stegall MD, Diwan T, Raghavaiah S, et al. Terminal complement inhibition decreases antibody-mediated rejection in sensitized renal transplant recipients. Am J Transplant. 2011;11:2405-2413.

39. Chapman JR, O'Connell PJ, Nankivell BJ. Chronic renal allograft dysfunction. J Am Soc Nephrol. 2005;16:3015-3026.

40. Sagoo P, Perucha E, Sawitzki B, et al. Development of a cross-platform biomarker signature to detect renal transplant tolerance in humans. J Clin Invest. 2010;120:1848-1861.

41. Newell KA, Asare A, Kirk AD, et al. Identification of a B cell signature associated with renal transplant tolerance in humans. J Clin Invest. 2010;120:1836-1847.

42. Sommerer C, Zeier M, Meuer S, Giese T. Individualized monitoring of nuclear factor of activated $\mathrm{T}$ cells-regulated gene expression in FK506-treated kidney transplant recipients. Transplantation. 2010;89: 1417-1423.
43. Glander P, Sommerer C, Arns W, et al. Pharmacokinetics and pharmacodynamics of intensified versus standard dosing of mycophenolate sodium in renal transplant patients. Clin J Am Soc Nephrol. 2010;5:503-511.

44. Bremer S, Mandla R, Vethe NT, et al. Expression of IMPDH1 and IMPDH2 after transplantation and initiation of immunosuppression. Transplantation. 2008;85:55-61.

45. Bohler T, Nolting J, Kamar N, et al. Validation of immunological biomarkers for the pharmacodynamic monitoring of immunosuppressive drugs in humans. Ther Drug Monit. 2007;29:77-86.

46. Kowalski RJ, Post DR, Mannon RB, et al. Assessing relative risks of infection and rejection: a meta-analysis using an immune function assay. Transplantation. 2006;82:663-668.

47. Holt DW. Therapeutic drug monitoring of immunosuppressive drugs in kidney transplantation. Curr Opin Nephrol Hypertens. 2002;11:657-663.

48. Clase CM, Mahalati K, Kiberd BA, et al. Adequate early cyclosporin exposure is critical to prevent renal allograft rejection: patients monitored by absorption profiling. Am J Transplant. 2002;2:789-795.

49. Undre NA, van Hoof J, Christiaans M, et al. Low systemic exposure to tacrolimus correlates with acute rejection. Transplant Proc. 1999;31:296-298.

50. van Gelder T, Hilbrands LB, Vanrenterghem Y, et al. A randomized double-blind, multicenter plasma concentration controlled study of the safety and efficacy of oral mycophenolate mofetil for the prevention of acute rejection after kidney transplantation. Transplantation. 1999;68:261-266.

51. Evans WE. Thiopurine S-methyltransferase: a genetic polymorphism that affects a small number of drugs in a big way. Pharmacogenetics. 2002;12:421-423.

52. Staatz CE, Tett SE. Clinical pharmacokinetics and pharmacodynamics of tacrolimus in solid organ transplantation. Clin Pharmacokinet. 2004;43:623-653.

53. Staatz CE, Tett SE. Pharmacokinetic considerations relating to tacrolimus dosing in the elderly. Drugs Aging. 2005;22:541-557.

54. Vadivel N, Garg A, Holt DW, Chang RW, Macphee IA. Tacrolimus dose in black renal transplant recipients. Transplantation. 2007;83: 997-999.

55. MacPhee IAM, Fredericks S, Tai T, et al. Tacrolimus pharmacogenetics: polymorphisms associated with expression of cytochrome P4503A5 and P-glycoprotein correlate with dose requirement. Transplantation. 2002;74:1486-1489.

56. Ng FL, Holt DW, Macphee IA. Pharmacogenetics as a tool for optimising drug therapy in solid-organ transplantation. Expert Opin Pharmacother. 2007;8:2045-2058.

57. Macphee IA. Use of pharmacogenetics to optimize immunosuppressive therapy. Ther Drug Monit. 2010;32:261-264.

58. Hesselink DA, van Schaik RH, van Agteren M, et al. CYP3A5 genotype is not associated with a higher risk of acute rejection in tacrolimus-treated renal transplant recipients. Pharmacogenet Genomics. 2008;18:339-348.

59. Thervet E, Loriot MA, Barbier S, et al. Optimization of initial tacrolimus dose using pharmacogenetic testing. Clin Pharmacol Ther. 2010;87:721-726.

60. Elens L, Bouamar R, Hesselink DA, et al. A new functional CYP3A4 intron 6 polymorphism significantly affects tacrolimus pharmacokinetics in kidney transplant recipients. Clin Chem. 2011;57: 1574-1583.

61. de Jonge H, Metalidis C, Naesens M, Lambrechts D, Kuypers DR. The P450 oxidoreductase $* 28 \mathrm{SNP}$ is associated with low initial tacrolimus exposure and increased dose requirements in CYP3A5-expressing renal recipients. Pharmacogenomics. 2011;12:1281-1291.

62. Passey C, Birnbaum AK, Brundage RC, Oetting WS, Israni AK, Jacobson PA. Dosing equation for tacrolimus using genetic variants and clinical factors. Br J Clin Pharmacol. 2011;72:948-957.

63. Lamba J, Lamba V, Schuetz E. Genetic variants of PXR (NR1I2) and CAR (NR1I3) and their implications in drug metabolism and pharmacogenetics. Curr Drug Metab. 2005;6:369-383. 
64. Press RR, Ploeger BA, den HJ, et al. Explaining variability in ciclosporin exposure in adult kidney transplant recipients. Eur J Clin Pharmacol. 2010;66:579-590.

65. Fredericks S, Jorga A, MacPhee IAM, et al. Multi-drug resistance gene-1 (MDR-1) haplotypes and the $C Y P 3 A 5 * 1$ genotype have no influence on ciclosporin dose requirements as assessed by $\mathrm{C} 0$ or $\mathrm{C} 2$ measurements. Clin Transplant. 2007;21:252-257.

66. Elens L, van Schaik RH, Panin N, et al. Effect of a new functional CYP3A4 polymorphism on calcineurin inhibitors dose requirements and trough blood levels in stable renal transplant patients. Pharmacogenomics. 2011;12:1383-1396.

67. Anglicheau D, Le Corre D, Lechaton S, et al. Consequences of genetic polymorphisms for sirolimus requirements after renal transplant in patients on primary sirolimus therapy. Am J Transplant. 2005;5:595-603.

68. Le Meur Y, Djebli N, Szelag JC, et al. CYP3A5*3 influences sirolimus oral clearance in de novo and stable renal transplant recipients. Clin Pharmacol Ther. 2006;80:51-60.

69. Miao LY, Huang CR, Hou JQ, Qian MY. Association study of ABCB1 and CYP3A5 gene polymorphisms with sirolimus trough concentration and dose requirements in Chinese renal transplant recipients. Biopharm Drug Dispos. 2007;29:1-5.
70. Picard N, Rouguieg-Malki K, Kamar N, Rostaing L, Marquet P. CYP3A5 genotype does not influence everolimus in vitro metabolism and clinical pharmacokinetics in renal transplant recipients. Transplantation. 2011;91:652-656.

71. van Schaik RH, van AM, de Fijter JW, et al. UGT1A9-275T>A/$2152 \mathrm{C}>\mathrm{T}$ polymorphisms correlate with low MPA exposure and acute rejection in $\mathrm{MMF} /$ tacrolimus-treated kidney transplant patients. Clin Pharmacol Ther. 2009;86:319-327.

72. Wang J, Yang JW, Zeevi A, et al. IMPDH1 gene polymorphisms and association with acute rejection in renal transplant patients. Clin Pharmacol Ther. 2007;83:711-717.

73. Crettol S, Venetz JP, Fontana M, et al. Influence of ABCB1 genetic polymorphisms on cyclosporine intracellular concentration in transplant recipients. Pharmacogenet Genomics. 2008;18:307-315.

74. Capron A, Mourad M, de MM, et al. CYP3A5 and ABCB1 polymorphisms influence tacrolimus concentrations in peripheral blood mononuclear cells after renal transplantation. Pharmacogenomics. 2010;11:703-714.
Pharmacogenomics and Personalized Medicine

\section{Publish your work in this journal}

Pharmacogenomics and Personalized Medicine is an international, peerreviewed, open access journal characterizing the influence of genotype on pharmacology leading to the development of personalized treatment programs and individualized drug selection for improved safety, efficacy and sustainability. This journal is indexed on the American Chemical

\section{Dovepress}

Society's Chemical Abstracts Service (CAS). The manuscript management system is completely online and includes a very quick and fair peer-review system, which is all easy to use. Visit http://www.dovepress. com/testimonials.php to read real quotes from published authors. 